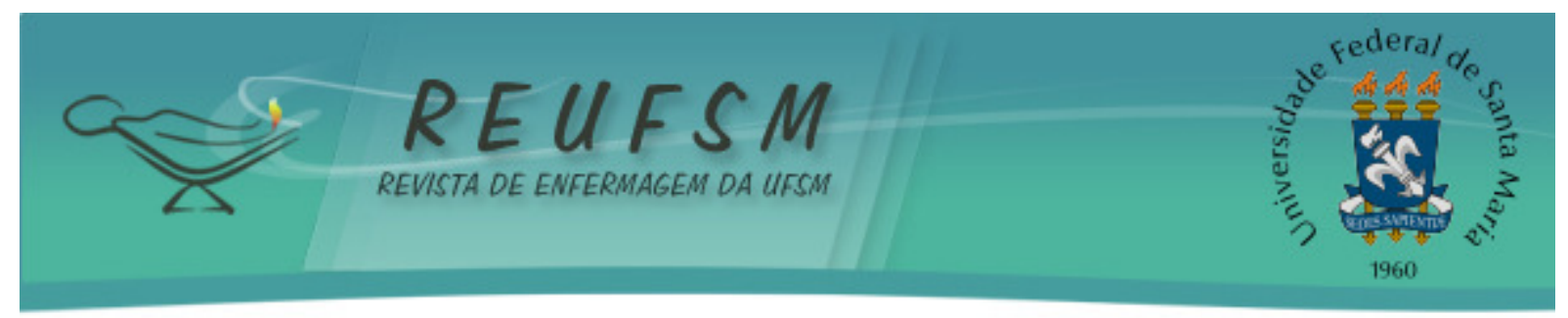

\title{
ARTIGO DE REFLEXÃO RISCOS PSICOSSOCIAIS EM ENFERMAGEM DE TERAPIA INTENSIVA: REFLEXÃO SOBRE POSSÍVEIS SOLUÇÕES
}

\author{
PSYCHOSOCIAL RISKS IN INTENSIVE CARE NURSING: REFLECTION ON \\ POSSIBLE SOLUTIONS
}

\section{RIESGOS PSICOSOCIALES EN ENFERMERÍA DE CUIDADOS INTENSIVOS: REFLEXIÓN SOBRE POSIBLES SOLUCIONES}

\author{
Jorge Luiz Lima da Silva ${ }^{1}$ \\ Gilvânia Barreto Feitosa Coutinho ${ }^{2}$ \\ Eliana Napoleão Cozendey da Silva ${ }^{3}$ \\ Liliane Reis Teixeira $^{4}$ \\ Karine Barreto Rodrigues da Silva ${ }^{5}$ \\ Rafael da Silva Soares ${ }^{6}$
}

Doi: $10.5902 / 2179769224494$

RESUMO: Objetivo: discutir estresse, burnout e possíveis soluções de enfrentamento do profissional de enfermagem atuante em unidades de terapia intensiva. Método: tratou-se de um estudo do tipo reflexão. Resultados: percebeu-se que o estresse laboral advém de três eixos principais: o ambiente da UTI, o processo de trabalho no ambiente em questão e também o estresse inerente à própria atividade do profissional de enfermagem. A partir dessas questões, tais fatores foram agrupados em institucionais, políticos e de qualidade de vida em ambiente de trabalho. Conclusão: são necessários esforço coletivo, condições políticas e aspectos institucionais favoráveis para melhorar a qualidade de vida do trabalhador. A resiliência e enfrentamento individual mostram-se insuficientes quando o ambiente laboral não é adequado.

Descritores: Saúde do trabalhador; Equipe de enfermagem; Unidades de terapia intensiva; Esgotamento profissional.

ABSTRACT: Aim: Discussing stress, burnout and possible solutions for coping with the nursing professional working in intensive care units. Method: this was a reflexive study. Results: it was noticed that the work stress comes from three main axes, the ICU setting, the work process in the environment in question and also the stress inherent in the activity itself of the nursing professional. From these issues, these factors were grouped into institutional, political and quality of life in the workplace, to elucidate. Conclusion: collective effort, political conditions and institutional aspects are necessary to improve the worker's quality of life. Individual resilience and coping are insufficient when the work environment is not adequate.

Descriptors: Occupational health; Nursing, team; Intensive care units; Burnout, professional.

RESUMEN: Objetivo: discurtir sobre el estrés, el agotamiento y las posibles soluciones de afrontamiento del profesional de enfermería que trabajan en unidades de cuidado intensivo.

\footnotetext{
${ }^{1}$ Professor adjunto. Doutor em Saúde Pública. Depto. Materno - infantil e Psiquiatria da Universidade Federal Fluminense / UFF.

${ }^{2}$ Doutoranda do Programa de Saúde Pública - Escola Nacional de Saúde Pública Ensp/ Fiocruz.

${ }^{3}$ Pesquisadora Visitante. Doutora em Saúde Pública - Escola Nacional de Saúde Pública Ensp/ Fiocruz.

${ }^{4}$ Pesquisadora. Doutora em Saúde Coletiva. Centro de Estudo e Ecologia Humana da Fundação Instituto Oswaldo Cruz / Fiocruz.

${ }^{5}$ Acadêmica de enfermagem - Universidade Federal Fluminense / UFF.

${ }^{6}$ Enfermeiro. Mestrando em Ciências do Cuidado em Saúde, Universidade Federal Fluminense / UFF.
} 


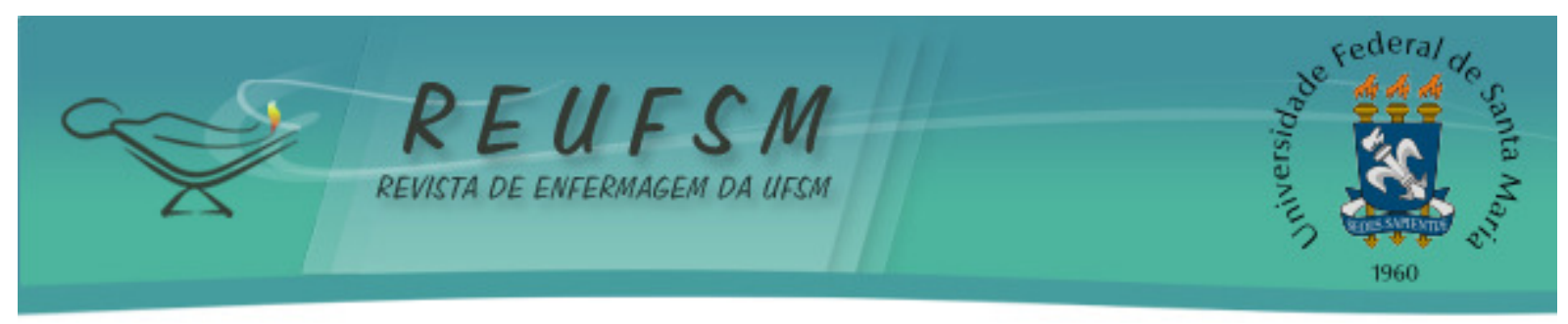

Método: se trata de un estudio reflexivo. Resultados: se observó que el estrés laboral tiene origen en tres ejes principales, el ambiente de la UCI, el proceso de trabajo en este tipo de espacio y también el estrés propio de la actividad del profesional de enfermería. A partir de esas cuestiones, tales fueron agrupados en institucionales, político y de calidad de vida en el ambiente de trabajo. Conclusión: es necesario esfuerzo colectivo, condiciones políticas e institucionales favorables para mejorar la calidad de vida de los trabajadores. Resiliencia y afrontamiento individual son insuficientes cuando el ambiente de trabajo no es adecuado.

Descriptores: Salud del trabajador; Equipo de enfermería; Unidades de cuidados intensivos; Agotamiento profesional.

\section{INTRODUÇÃO}

O estresse relacionado ao ambiente de trabalho, ou estresse ocupacional, pode ser definido como as respostas físicas e emocionais prejudiciais que ocorrem quando as exigências do trabalho superam as capacidades, recursos ou necessidades do trabalhador. ${ }^{1-2}$ Robert Karasek foi um dos pioneiros a relacionar dois fatores importantes referentes ao trabalho: as relações sociais do ambiente laboral (consideradas fontes causadoras de estresse); e consequências físicas e psicológicas para saúde (denominados, atualmente, como aspectos psicossociais). O desequilíbrio nestas dimensões pode favorecer a exacerbação do estresse e trazer consequências negativas à saúde do trabalhador. ${ }^{1,3}$

Certos profissionais, como os de enfermagem, são mais expostos aos agentes estressores mediante a complexidade das atividades desenvolvidas e ambiente laboral no qual estão inseridos. ${ }^{4}$ Alguns exemplos das dificuldades encontradas por essa categoria são: sobrecarga de trabalho; jornada e turnos de trabalho extenuantes; mudanças constantes de setor; e carga psíquica. Estes fatores são intensificados nos setores fechados e isolados como as unidades de terapia intensiva (UTI). ${ }^{1}$

O termo burnout foi citado na literatura em 1953, em estudo de caso de Schwartz e Will, denominado Miss Jones, no qual foi descrita a problemática de uma enfermeira psiquiátrica desiludida com o seu trabalho. Naquela época, os autores descreveram os sintomas da paciente como psicológicos, físicos e emocionais. A síndrome possui conceito tridimensional que articula três fatores básicos que podem aparecer em conjunto ou independentes que são: exaustão emocional, despersonificação e baixa realização pessoal., ${ }^{1,5-8}$

Há de se refletir sobre a qualidade de vida no ambiente de trabalho, o que envolve propiciar condições para o maior desempenho, bem como condições básicas de atuação dos profissionais e a implementação de estratégias para o enfrentamento do estresse ocupacional. 


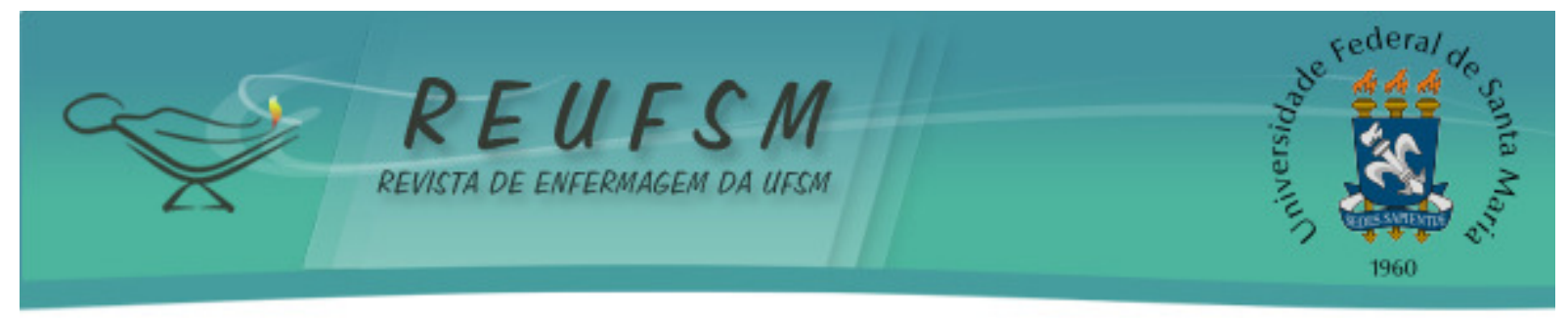

Assim sendo, questiona-se é possível traçar estratégias de amenização ou prevenção do estresse e do burnout em UTI?

Este trabalho objetiva discutir estresse, burnout e possíveis soluções de enfrentamento do profissional de enfermagem atuante em UTI.

\section{MÉTODO}

A reflexão compõe um dos artigos da tese de doutoramento defendida na Fundação Instituto Oswaldo Cruz em 2015: "aspectos psicossociais e síndrome de burnout entre trabalhadores de enfermagem intensivistas". O presente estudo apresenta dois eixos temáticos de discussão: O trabalho na UTI, ambiente, estresse laboral e burnout abordando, mais detalhadamente, os conceitos e suas repercussões no trabalho do profissional de enfermagem e posteriormente, os Desafios e possíveis propostas de enfrentamento.

\section{O trabalho na UTI, ambiente, estresse laboral e burnout}

No ambiente nosocomial, a atenção tem se voltado para os ambientes de UTI, setor fechado, onde são realizados cuidados aos pacientes críticos. Neste local, há importante relação entre a organização do trabalho e estresse, principalmente, para os trabalhadores da enfermagem. ${ }^{9}$

Grande parte das instituições hospitalares brasileiras utiliza o modelo americano para o dimensionamento do pessoal de enfermagem, inclusive na UTI, por meio da fórmula proposta pela Liga Nacional de Educação em Enfermagem dos Estados Unidos da América do Norte e pela Associação Americana de Enfermeiros. Esse modelo é baseado nas horas dedicadas a cada tipo de paciente e leva em conta a realidade norte-americana de prestação de serviços de saúde, em que há mais recursos humanos, físicos e tecnológicos distintos da realidade de países subdesenvolvidos e emergentes. ${ }^{10} \mathrm{~A}$ insuficiência de profissionais intensivistas no sistema de saúde americano tem sido discutida, demonstrando que o aumento das demandas não foi acompanhado pelo incremento de recursos físicos, tecnológicos e humanos. ${ }^{11}$

No Brasil, a resolução n. 26 de 11/05/2012, da Agência Nacional de Vigilância Sanitária (Anvisa) modifica a relação de um enfermeiro por oito pacientes, para um enfermeiro por dez pacientes; além de retirar um técnico de apoio assistencial por turno. Tal medida foi imposta sem negociação com a classe de trabalhadores. Na prática, a equipe passa a ser composta por menos profissionais de enfermagem para lidar diretamente com doentes em estado crítico. 


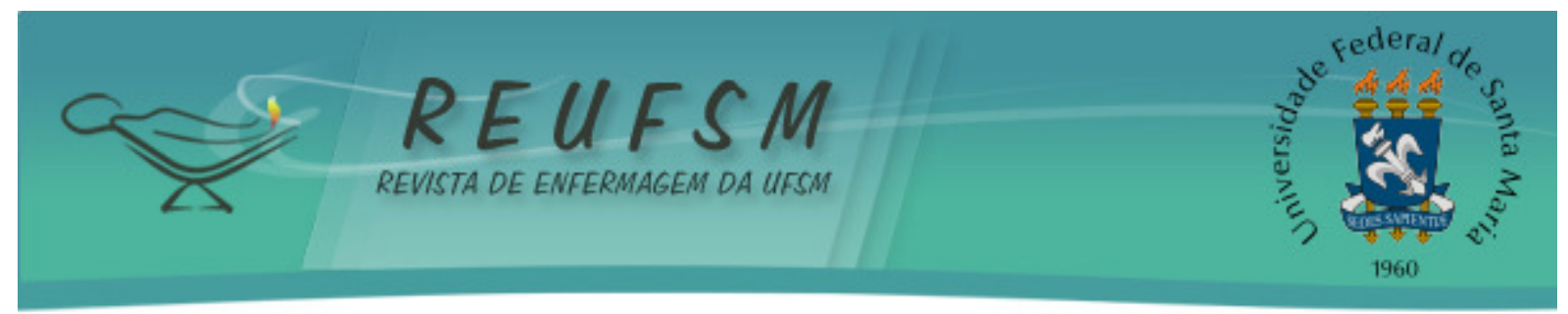

O Conselho Federal de Enfermagem (Cofen) aponta a necessidade de ingressar com ações judiciais para que sejam respeitadas condições implementadas em 2010, pois as medidas atuais favorecem o capital e a perda da qualidade de assistência, impondo nova organização do trabalho e conduzido ao desgaste profissional. A carga horária também vem sendo discutida e é considerada penosa, desgastante e com baixo retorno financeiro para o trabalhador. Diante desse fato, para se manter a qualidade de vida, é necessário um segundo e/ou terceiro vínculo, que resulta em aumento da jornada de trabalho e redução do tempo livre para a vida pessoal e social. ${ }^{12}$ Estes últimos elementos integram o apoio social que vêm sendo negligenciado, apesar de citado em estudos como amenizador natural do estresse. ${ }^{13-14}$

O trabalho em equipe é fundamental para a colaboração mútua, o qual estimula o enfrentamento dos desafios do cotidiano laboral. ${ }^{15-18}$ Outras insatisfações com o trabalho estão relacionadas às questões como reuniões com a chefia, críticas dos subordinados, sentirse só nas tomadas de decisões, receio de perder o emprego, baixos salários e sentir-se desvalorizado. Um possível quadro de burnout pode ser observado diante da presença contínua de insatisfação do enfermeiro com a sua atividade profissional, interferindo em sua saúde e qualidade de vida. ${ }^{19}$

A síndrome de burnout (SB) possui quatro níveis de evolução: 1) falta de vontade, ânimo ou prazer de ir trabalhar, dores nas costas, pescoço e coluna; 2) deterioração do relacionamento do profissional com outros, com sensação de perseguição, aumento do absenteísmo e da rotatividade de empregos; 3) diminuição notável da capacidade ocupacional, início de doenças psicossomáticas tais como alergias, psoríase, picos hipertensivos dentre outras; 4) alcoolismo, drogadição, ideias ou tentativas de suicídio. ${ }^{20}$

Estudo internacional identificou 34,9\% de profissionais de enfermagem afetados pela síndrome de burnout. Também revelou, por análise de regressão logística, que o tempo e o ambiente de trabalho foram significativamente associados à insatisfação com o trabalho e SB entre profissionais de enfermagem. Por meio de coeficiente de correlação de Pearson, evidenciou-se que a insatisfação no trabalho apresentou uma correlação efetiva com burnout e a equipe de enfermagem. ${ }^{21}$

De acordo com a Organização Mundial de Saúde (OMS) e a Organização Internacional do Trabalho (OIT), o fenômeno globalização é um grande responsável pelo aumento de incidência de doenças ocupacionais e acidentes de trabalho. $\mathrm{O}$ aumento da carga de trabalho e o 


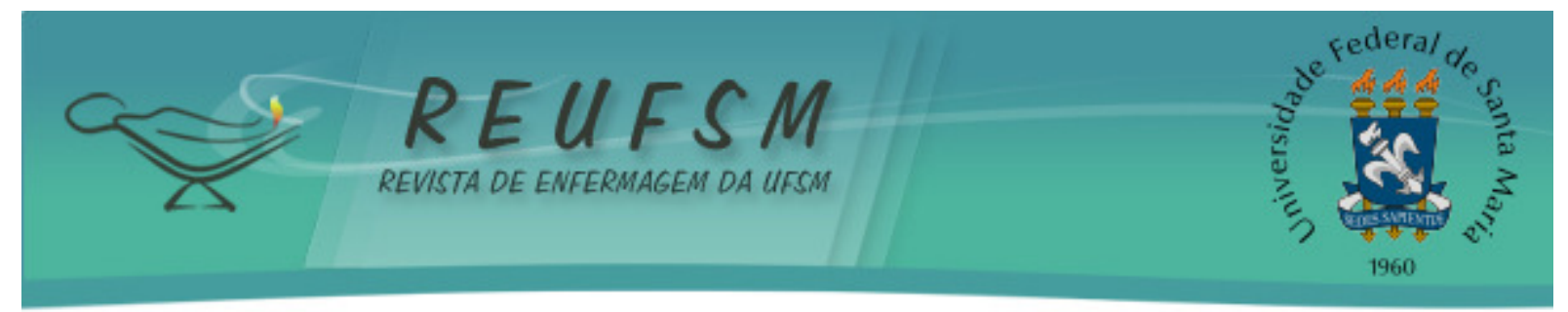

ritmo de vida cada vez mais acelerado, impuseram ao trabalhador uma nova realidade para a qual o mesmo não estava preparado. Ainda, segundo a OIT, os impactos negativos do estresse são multiformes, podem incluir doenças cardíacas e gastrintestinais, bem como problemas psicossomáticos e psicossociais, os quais possuem forte influência do ambiente laboral, pois o grau de estresse ao qual o trabalhador é submetido está relacionado ao nível de demanda e controle sobre o trabalho. Esses por sua vez, em desequilíbrio, podem favorecer o aumento de

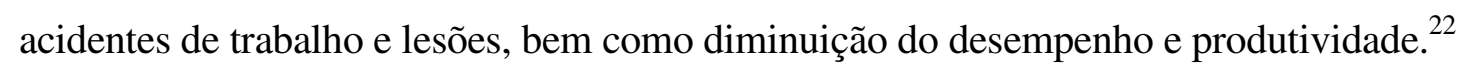

\section{Desafios e possíveis propostas de enfrentamento}

Há de se pensar que as modificações no setor saúde para os trabalhadores discutidos neste ensaio sejam em âmbito político, institucional e pautados na qualidade de vida. Entende-se que somente ações articuladas e, ao mesmo tempo, sinérgicas, poderão ir contra o paradigma vigente.

Mas apesar dos avanços, pergunta-se: seria suficiente? Há uma inter-relação entre as instituições envolvidas para que o trabalhador seja, enfim, favorecido? Haverá interesse político e econômico para que se implementem essas mudanças?

$\mathrm{Na}$ investigação das relações saúde-trabalho-doença, o trabalhador de enfermagem deve ser ouvido, seja individual ou coletivamente, pois diante dos avanços e da sofisticação das técnicas para o estudo dos ambientes e condições de trabalho, há de se reconhecer que estes profissionais, cotidianamente, vivenciam condições e circunstâncias diversas, imprevistos e improvisos. Neste caso, são capazes de descrever essas situações e explicar a relação do trabalho com o adoecimento, sob sua ótica. ${ }^{23}$

Diante do exposto, observa-se mais uma vez situação contraditória. Será que as empresas que são hospitais, hospitais-escola e hospitais de grande porte poderiam investir na qualidade de vida de seus profissionais de cuidados intensivos? Conferência Internacional sobre o Trabalho, realizada em agosto de 2013 em Helsinki na Finlândia, reconhece que o bem-estar no trabalho é essencial para a qualidade de vida dos trabalhadores e para a produtividade em qualquer área de atuação. ${ }^{24}$

Outra pesquisa visou intervir na organização do trabalho, após avaliar a qualidade de sono e verificar a presença de sonolência diurna excessiva entre profissionais de enfermagem dos plantões noturnos das UTI do Instituto Central do Hospital das Clínicas da Faculdade de Medicina da Universidade de São Paulo. O estudo demonstrou que 97,3\% dos profissionais apresentaram 


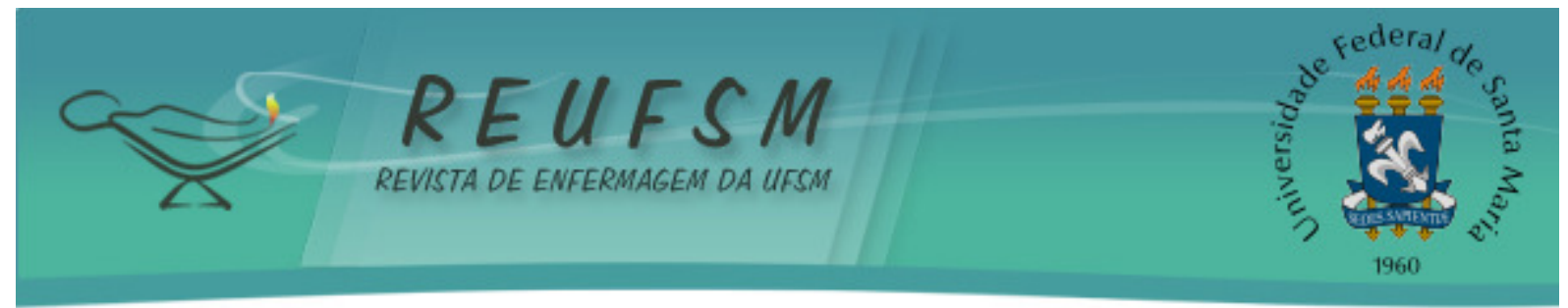

má-qualidade do sono e 70,6\% sonolência diurna excessiva. Algumas intervenções relacionadas às causas do problema foram necessárias. Realizou-se planejamento e organização do local, como: sala de conforto com televisão, rádio e camas adequadas; rotina de rodízio para o descanso e alimentação; e a mudança da carga horária do turno noturno, alterando a jornada de doze horas para oito horas e pausas de 15 minutos, a cada duas horas de trabalho. ${ }^{25}$

Algumas ações apontam para uma sinergia de fatores que incluem os aspectos políticos os quais trazem o âmbito mais geral e de negociação; os institucionais abarcam características que envolvem aspectos sociais e de gestão; e qualidade de vida no trabalho que enumera ações pontuais para alcançar esse propósito.

Quadro 1: Ações pertinentes para mudança no ambiente de trabalho em UTI.

\begin{tabular}{|c|c|}
\hline ASPECTOS & AÇÕES \\
\hline Políticos & $\begin{array}{l}\text {-Discussões amplas sobre a carga horária semanal; }{ }^{1} \\
\text {-Incentivo ao interesse da classe trabalhadora por meio de movimentos } \\
\text { políticos; }{ }^{1,13,18} \\
\text {-Construção de uma rede nacional de negociação junto aos sindicatos, conselhos e } \\
\text { governo federal; }{ }^{1} \\
\text {-Discussão sobre os avanços em níveis estaduais e locais para reflexão e possível } \\
\text { implementação em nível nacional; } \\
\text {-Ações implementadas em nível nacional, as quais visem fortalecer a classe } \\
\text { trabalhista na federação; } \\
\text {-Criação e implementação de uma política de qualidade de vida no trabalho para } \\
\text { trabalhadores do setor saúde. }\end{array}$ \\
\hline Institucionais & $\begin{array}{l}\text {-Implantação de uma gestão mais participativa, de forma a promover maior } \\
\text { percepção do controle sobre o trabalho; }{ }^{1,4,6,13,18,19,21} \\
\text {-Identificação de fatores que possam conduzir a riscos psicossociais à saúde do } \\
\text { trabalhador no setor e amenizá-los; }{ }^{1,3,4,9,18} \\
\text {-Implementação de programas de atendimento aos trabalhadores direcionados para } \\
\text { suprir as necessidades apontadas pelas equipes de cada setor; } \\
\text {-Estabelecer metas que envolvam também a qualidade do cuidado, com o } \\
\text { monitoramento dos recursos necessários, de forma a evitar o desgaste físico e } \\
\text { mental do profissional; }{ }^{1,4,18,19} \\
\text {-Instruição da capacitação como atividades de trabalho, incluindo nas horas } \\
\text { remuneradas ao trabalhador e, sempre que possível, investimento a qualificação e } \\
\text { sensibilização quanto aos riscos psicossociais; } \\
\text {-Manutenção do número adequado de profissionais de acordo com as demandas e } \\
\text { número dos pacientes; }{ }^{3,9,15,18,21} \\
\text {-Revisão de valores atribuídos ao capital, em detrimento da qualidade do trabalho e } \\
\text { consequentemente à vida humana; } \\
\text {-Estimulo a comunicação mais efetiva entre os membros da equipe de } \\
\text { enfermagem; }{ }^{3,4,10,19} \\
\text {-Melhorias das condições físicas do ambiente de trabalho; } \\
\text {-Mudanças na forma de reconhecimento e valorização do funcionário, seja de } \\
\text { maneira formal (escrita) ou informal, por meio de expressão verbal de elogios, } \\
\text { tornando o convívio mais humanizado;, } \\
\text {-Estímulo a maior autonomia ao trabalhador. }\end{array}$ \\
\hline
\end{tabular}




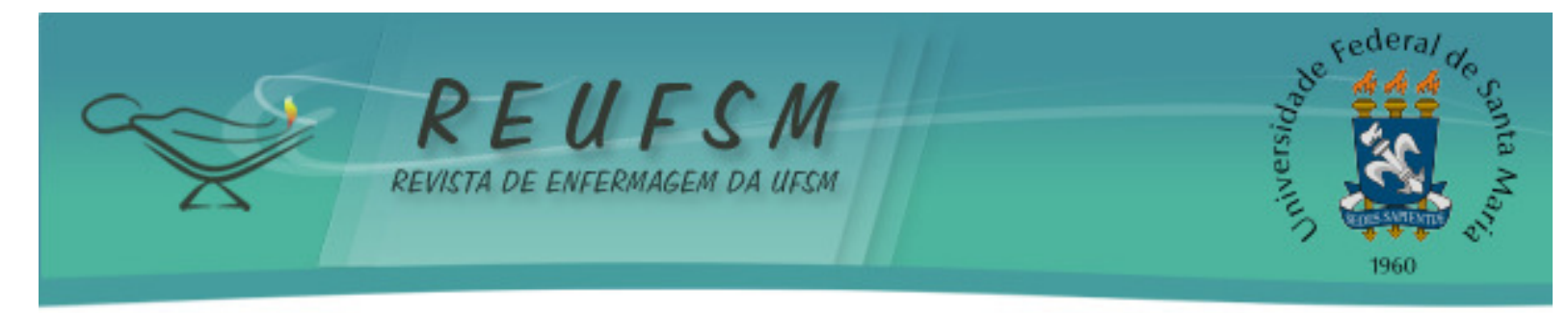

\begin{tabular}{|c|c|}
\hline $\begin{array}{llll}\text { Qualidade } & \text { de } & \text { vida } & \text { no } \\
\text { trabalho } & & & \end{array}$ & $\begin{array}{l}\text {-Instituição do acompanhamento psicológico dos profissionais; } \\
\text {-Implementação de técnicas de relacionamento no ambiente de trabalho, como } \\
\text { estratégia de amenização do estresse: ginástica laboral, encontros sociais formais } \\
\text { para motivação e escuta das demandas da equipe; pequenas reuniões mensais para } \\
\text { discussão de pontos considerados críticos pela equipe, desde que acordado com o } \\
\text { grupo; } \\
\text {-Obrigatório o repouso no ambiente de trabalho, com pausas periódicas - } \\
\text { respeitando a legislação local; } \\
\text {-Estimulo ao bom relacionamento interpessoal, através de reuniões de equipe } \\
\text { constante, com levantamento de problemas e possíveis soluções de forma a ouvir } \\
\text { um grupo;,19 } \\
\text {-Promoção de encontros informais para propiciar o relaxamento, em especial em } \\
\text { datas comemorativas, com o envolvimento dos familiares e pacientes, quando } \\
\text { possível. }\end{array}$ \\
\hline
\end{tabular}

O Quadro 1 representa a construção do esforço coletivo e necessário para que as organizações ouçam as reivindicações dos seus trabalhadores, como um elemento motriz do processo de trabalho. As condições políticas devem ser favoráveis, e seriam o alicerce para os demais itens, dessa forma, perpassando pelos aspectos institucionais que favoreceriam, por sua vez, os elementos de qualidade de vida. Com o tempo, as ações passariam a ser sinérgicas e interdependentes. Com isso, retira-se o foco do enfrentamento individual o qual se apresenta desgastado e insuficiente. Só pode haver resiliência e enfrentamento da pessoa, caso encontre ambiente laboral propício para essa dinâmica.

Surge um debate social que tem como foco a possibilidade de organização, a partir dos locais de trabalho, de forma a possibilitar discussão das demandas de maneira democrática e igualitária. Com isso, permite-se a participação do trabalhador, visando a análise das questões ligadas à qualidade de vida no trabalho e à defesa da saúde e da vida. ${ }^{14}$ Além disso, devem ser consideradas as variações políticas, geográficas, institucionais e ambientais, tendo em vista as mudanças a serem implementadas em prol do trabalhador de enfermagem. Existem especificidades e demandas singulares por parte da equipe de enfermagem pelas regiões do Brasil e do mundo, mesmo quando se foca a atenção do estudo em unidades de cuidados intensivos.

Seria muito importante que os profissionais pudessem usufruir de tais recursos para aumentar seu grau de resiliência. O avanço na discussão e implementação desses aspectos é relevante para a saúde do trabalhador e para as organizações, pois fortalece a credibilidade do serviço, que se pautaria em preceitos de qualidade e de respeito aos profissionais intensivistas. 


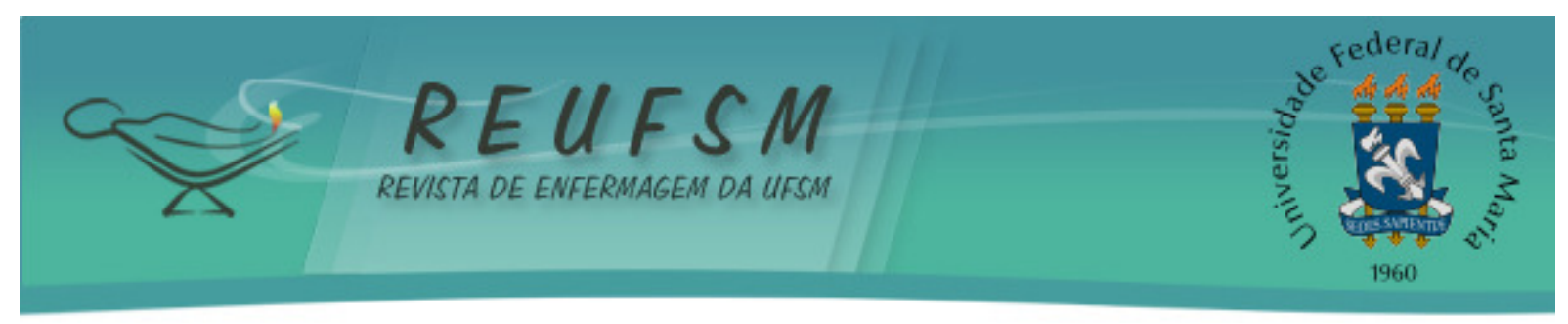

\section{CONCLUSÃO}

Mediante a reflexão deste estudo teórico, pode-se constatar que há necessidade de atenção à saúde do trabalhador que exerce suas atividades em ambiente de terapia intensiva, de forma que os aspectos políticos, institucionais e sociais possam proporcionar qualidade de vida no trabalho. Tal processo deverá se desenvolver de forma sinérgica e com participação efetiva dos intensivistas envolvidos.

É importante frisar que há grande entrave ditado pelo modelo econômico neoliberal que prejudica ações voltadas para qualidade de vida no trabalho no Brasil. As instituições possuem recursos escassos para programas dessa natureza, na área de saúde do trabalhador.

Em contrapartida, tais iniciativas devem ser promovidas, pois o labor atua como um dos elementos responsáveis pela gênese das desordens mentais, e está inserido no processo saúde-doença do trabalhador. Nota-se também que existe considerável contribuição dos estudos científicos na área, os quais devem receber mais atenção pelas instituições envolvidas, bem como medidas estratégicas para o acesso livre a esse material, o que contribuiria para o processo de gestão, fiscalização do trabalho e saúde dos profissionais.

\section{REFERÊNCIAS}

1. Lima Silva JL, Campos DA, Teixeira LR. Discussão sobre as causas da síndrome de burnout e suas implicações à saúde do profissional de enfermagem. Aquichán. 2012 ago;12(2):144-59.

2. Wright K. Alleviating stress in the workplace: advice for nurses. Nurs Stand. 2014;28(20):37-42.

3. Souza IAS, Pereira MO, Oliveira MAF, PPH, Gonçalves RMDA. Processo de trabalho e seu impacto nos profissionais de enfermagem em serviço de saúde mental. Acta Paul Enferm. 2015;28(5):447-53.

4. Moustaka E, Constantinidis TC. Sources and effects of work-related stress in nursing. J Health Sci. 2010;4(4):210-6.

5. Barlem ELD, Lunardi V L, Lunardi G L, Tomaschewski-Barlem JG, Almeida AS, Hirsch CD. Psycometric characteristics of the moral distress scale in Brazilian nursing professionals. Texto \& Contexto Enferm. 2014;23(3):563-72.

6. Oliveira V, Pereira T. Ansiedade, depressão e burnout em enfermeiros: impacto do trabalho por turnos. Rev Enferm Referência. 2012;serIII(7):43-54.

7. Teixeira FG, Silva MRS, Medeiros GL. Síndrome de burnout - a interface entre o trabalho na área da educação e na enfermagem. Referência. 2010;serIII(2):101-9. 


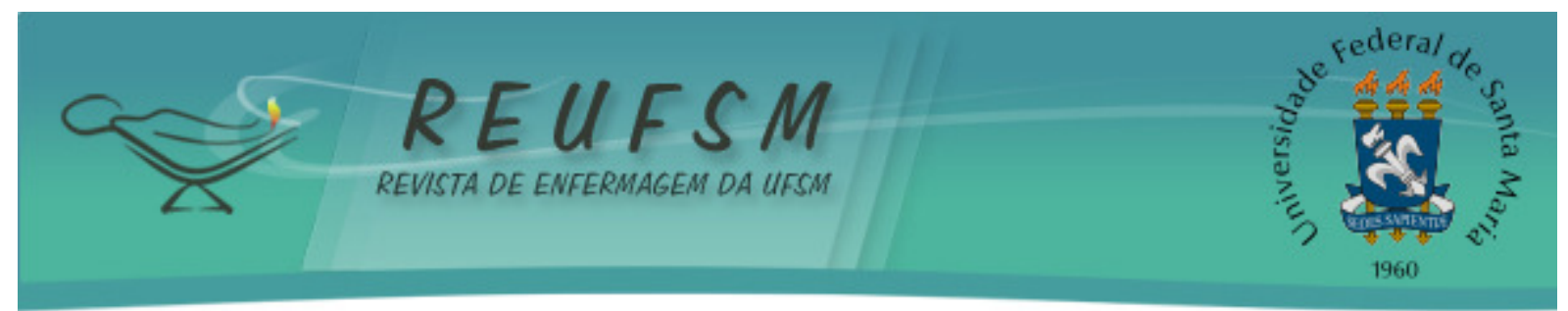

8. Murofuse NT, Abranches SS, Napoleão AA. Reflexões sobre estresse e burnout e a relação com a enfermagem. Rev Latinoam Enferm. 2005;13(2):255-61.

9. Monte PF, Lima FET, Neves FMO, Studart RMB, Dantas RT. Estresse dos profissionais enfermeiros que atuam na unidade de terapia intensiva. Acta Paul Enferm. 2013;26(5):421-7.

10. Portero de la Cruz S, Vaquero Abellán M. Professional burnout, stress and job satisfaction of nursing staff at a university hospital. Rev Latinoam Enferm. 2015;23(3):543-52.

11 Panunto MR, Guirardello EB. Professional nursing practice: environment and emotional exhaustion among intensive care nurses. Rev Latinoam Enferm. 2013;21(3):765-72.

12. Schmidt DRC, Paladini M, Biato C, Pais JD, Oliveira AR. Qualidade de vida no trabalho e burnout em trabalhadores de enfermagem de unidade de terapia intensiva. Rev Bras Enferm. 2013;66(1):13-7.

13. Dalmolin GL, Lunardi VL, Barlem ELD, Silveira RS. Implicações do sofrimento moral para os (as) enfermeiros (as) e aproximações com o burnout. Texto \& Contexto Enferm. 2012;21(1):200-8.

14. International Labour Office (ILO). Stress prevention at work checkpoints: practical improvements for stress prevention in the workplace. Geneva: ILO; 2012.

15. Andolhe R, Barbosa RL, Oliveira EM, Costa ALS, Padilha KG. Estresse, coping e burnout da equipe de enfermagem de unidades de terapia intensiva: fatores associados. Rev Esc Enferm USP. 2015;49(N Esp):58-64.

16. Lima Silva JL, Soares RS, Costa FS, Ramos DS, Lima FB, Teixeira LR. Fatores psicossociais e prevalência da síndrome de burnout entre trabalhadores de enfermagem intensivistas. Rev Bras Ter Intensiva. 2015;27(2):125-33.

17. Borges EMN, Ferreira TJR. Relaxamento: estratégia de intervenção no stress. Rev Port Enferm Saúde Mental. 2013;(10):37-42.

18. Barlem ELD, Lunardi VL, Lunardi GL, Tomaschewski-Barlem JG, Silveira RS, Dalmolin GL. Moral distress in nursing personnel. Rev Latinoam Enferm. 2013;21(N Esp):79-87.

19. Monteiro JK, Oliveira ALL, Ribeiro CS, Grisa GH, Agostini N. Adoecimento psíquico de trabalhadores de unidades de terapia intensiva. Psicol Cienc Prof. 2013;33(2):366-79.

20. Pereira AM, Queirós C, Gonçalves SP, Carlotto MS, Borges E. Burnout e interação trabalho-família em enfermeiros: estudo exploratório com o Survey Work-Home Interaction Nijmegen (SWING). Rev Port Enferm Saúde Mental. 2014;(11):24-30.

21. Zhou W, He G, Wang H, He Y, Yuan Q, Liu D. Job dissatisfaction and burnout of nurses in Hunan, China: a cross-sectional survey. Nurs Health Sci. 2015;17(4):444-50.

22. Schmidt DRC, Paladini M, Biato C, Pais JD, Oliveira AR. Qualidade de vida no trabalho e burnout em trabalhadores de enfermagem de unidade de terapia intensiva. Rev Bras Enferm. 2013;66(1):13-7.

23. Roloff DIT, Cezar-Vaz MR, Bonow CA, Lautert L, Sant'Anna CF, Couto AM. Enfermeiros do trabalho: experiência interdisciplinar em saúde do trabalhador. Rev Bras Enferm. 2016;69(5):897-905. 


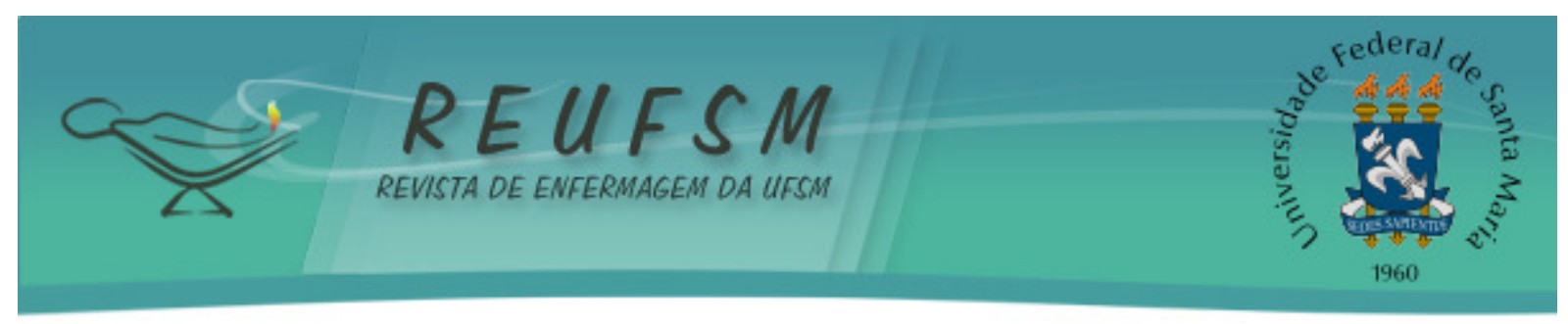

24. European Agency for Safety and Health at Work. Well-being at work: creating a positive work environment. Luxembourg: Publications Office of the European Union; 2013.

25. Coelho MP, Pinto OO, Mota MC, Crispim CA. Prejuízos nutricionais e distúrbios no padrão de sono de trabalhadores da Enfermagem. Rev Bras Enferm. 2014;67(5):832-42.

Data de submissão: 19/10/2016

Data de aceite: $24 / 08 / 2017$

Autor correspondente: Jorge Luiz Lima da Silva

Endereço: Rua Dr. Celestino, 74- sala 51/ Centro, Niterói-RJ

CEP: 24020-091

E-mail: jorgeluizlima@gmail.com 